\title{
EXPLORATORY SPATIAL DATA ANALYSIS OF REGIONAL ECONOMIC DISPARITIES IN BEIJING DURING 2001-2007
}

\author{
Xiaoyi Ma*, Tao Pei \\ State Key Laboratory of Resources and Environmental Information System, Institute of Geographic Sciences and \\ Natural Resources Research, CAS, 100101 Beijing, China - (maxy, peit)@lreis.ac.cn
}

\begin{abstract}
KEY WORDS: Regional Economy, The Olympic Games, Exploratory Spatial Data Analysis, GIS, Spatial Autocorrelation, Spatial Heterogeneity
\end{abstract}

\begin{abstract}
:
The unbalanced development of regional economics in Beijing has induced the ubiquitous regional economic disparities and may affect the sustainable development of economy and social stability. How to alleviate this unbalanced status has been an important issue which the society and government face. As usual, the hold of Olympics Games may boost the economy of host city, however it is not clear that the event could narrow the regional economic disparities. In order to investigate the influence of the 2008 Olympics Games for the development of regional economics in Beijing, this paper studies the space-time dynamics of economic development of Beijing since the year when Beijing won the bid (the year of 2001) using exploratory spatial data analysis (ESDA), and based on the county level and measure index of the regional per capita gross domestic product (GDP). The results show not strong evidences of global spatial autocorrelation, but clear evidences of local spatial autocorrelation and spatial heterogeneity in the distribution of regional per capita GDP. Driven by the bid winning of 2008 Olympics, the total economic disparity in Beijing had not been alleviated, and the situation got even more complicated. Since the economic increasing-speeds of Changping and Shijingshan Districts were significantly lower than their some neighbouring regions, a new centre-surrounding polarization scheme was gradually replacing the North-South polarization scheme in Beijing from 2001 to 2007.
\end{abstract}

\section{INTRODUCTION}

The unique history foundation and location condition cause the unbalanced development of regional economics in Beijing. This unbalanced status induces the ubiquitous regional economic disparities and may affect the sustainable development of economy and social stability. How to alleviate this unbalanced status has been an important issue which the society and government face. As history had proved that, the hold of Olympics may boost the economy of host city. The 2008 Olympic Games provided Beijing with an opportunity to successfully promote the economic development and accelerate the completion of major infrastructure upgrades in public service industry, transportation and other sectors. According to the research report of Beijing Municipal Statistic Bureau, the total investment for the 2008 Olympic Games from 2002 to 2007 was about 351 hundred million dollars, which efficiently promoted the growth in GDP of Beijing. In 2006, the Beijing's per capita gross regional product reached 6,210 dollars exceeding the target of 6000 dollars at the end of 2008, two years ahead of previously predicted. However, whether or not the Olympic Games could lessen the regional economic disparities is unclear.

In order to investigate the influence of the 2008 Olympic Games for the development of regional economic disparities in Beijing, this paper studies the space-time dynamics of economic development of Beijing since the year 2001 when Beijing won the bid, using exploratory spatial data analysis (ESDA). ESDA emphasizes the significance of spatial interactions and geographical location in the studies of regional economic development. By identifying spatial autocorrelation and spatial heterogeneity, the economic performance can be characterized according to time. Therefore, ESDA is a powerful tool for revealing the development of regional economic disparities. Several previous studied have been implemented on this issue focusing on the EU regions. Le Gallo and Ertur (2003), López Bazo et al. (2004) applied ESDA to study the distribution of regional per capita GDP in Europe. Ertur and Koch (2006) investigated the case affected by the enlargement of the European Union from 1995 to 2000. In addition, there are some studies have assessed the regional economic disparities using ESDA in China, including the work of Pu et al. (2005) on the Jiangsu province and Qiu et al. (2009) on the Huaihai economic zone of China. However, few of them revealed the space-time dynamics of regional economics caused by important historical events or national policy guides in China.

Consequently, this study, combining ESDA with GIS technology, investigates the development of regional economics after 2001, and attempts to explore the possibility that important historical events or national policy guides may associate with change in spatial patterns of regional economic disparities over time. Our method is based on the county level and measure index of the regional per capita gross domestic product (GDP).

\section{MATHODOLOGY AND DATA}

\subsection{Exploratory spatial data analysis (ESDA)}

Exploratory spatial data analysis (ESDA) is a set of techniques to describe and visualize spatial distributions, discover patterns of spatial association (spatial clustering or hot spots), identify atypical observations (outliers), and suggest different spatial regimes or other forms of spatial heterogeneity (Anselin, 1994;

\footnotetext{
* Corresponding author. Email: maxy@lreis.ac.cn.
} 
1996; 1999). Central to ESDA is the measure of global and local spatial autocorrelation.

The measure of global spatial autocorrelation is usually based on Moran's I statistic, expressed as:

$$
I=\frac{n}{\sum_{i=1}^{n}\left(x_{i}-\bar{x}\right)^{2}} \frac{\sum_{i=1}^{n} \sum_{j=1}^{n} w_{i j}\left(x_{i}-\bar{x}\right)\left(x_{j}-\bar{x}\right)}{\sum_{i=1}^{n} \sum_{j=1}^{n} w_{i j}}
$$

where $\boldsymbol{n}$ is the number of regions in the study area, $\boldsymbol{x}_{\boldsymbol{i}}$ and $\boldsymbol{x}_{\boldsymbol{j}}$ are the attribute values for region $\boldsymbol{i}$ and $\boldsymbol{j}, \overline{\boldsymbol{x}}$ is the mean of the attribute value for the $\boldsymbol{n}$ regions, and $\boldsymbol{w}_{\boldsymbol{i j}}$ is the spatial weight matrix, which is usually defined as a bivariate symmetric matrix $W$ to express the spatial contiguity relationship of regions. This paper adopts the spatial weight matrix based on the distance between the centroids of regions, that is, the corresponding element $\boldsymbol{w}_{\boldsymbol{i j}}=1$ if the distance between regions $\boldsymbol{i}$ and $\boldsymbol{j}$ is within the threshold distance and zero otherwise.

The Moran's I statistic is based on the measure of covariance $\left(x_{i}-\bar{x}\right)\left(x_{j}-\bar{x}\right)$, which is used to assess the similarity of specified attribute values of variable $\boldsymbol{X}$ (Getis and Ord, 1996). The inference of significance can be based on the permutation approach (Anselin, 1995).

Moran's I statistic can be visualized as the slope in the Moran scatter plot (Anselin, 1995), which plots the spatially lagged variable $(\boldsymbol{W} \boldsymbol{y})$ on the original variable $(\boldsymbol{y})$, here the $\boldsymbol{y}$ are in deviations from their mean. The four different quadrants of the Moran scatter plot represent the four types of local spatial association respectively. The upper right $(\mathrm{HH})$ and the lower left (LL) quadrants represent positive spatial autocorrelation, that is, a region with a high (low) value surrounded by regions also with high (low) values. The upper left (LH) and lower right (HL) quadrants correspond to negative spatial autocorrelation, i.e., a region is surrounded by regions with dissimilar values.

As one of the local spatial autocorrelation tools, the Moran scatter plot can identify local spatial clustering with high or low values, and indicate what patterns of spatial association make more contribution for the results of global spatial autocorrelation statistic, and visualize atypical observations. It makes up the major shortcoming of global spatial autocorrelation statistic, which can only indicate overall clustering. However, the Moran scatter plot does not indicate significance.

Local indicators of spatial autocorrelation (LISA) defined by Anselin (1995) provides a way to assess significant local spatial patterns, besides it can be used similarly to the Moran scatter plot.

The local Moran's statistics is a familiar LISA formulated as the follows:

$$
I_{i}=\frac{\left(x_{i}-\bar{x}\right)}{m_{0}} \sum_{j} w_{i j}\left(x_{i}-\bar{x}\right) \text { with } m_{0}=\sum_{i} \frac{\left(x_{i}-\bar{x}\right)^{2}}{n}
$$

where $\boldsymbol{n}$ is the number of regions in the study area, $\boldsymbol{x}_{\boldsymbol{i}}$ is the attribute values for region $\boldsymbol{i}, \overline{\boldsymbol{x}}$ is the mean of the attribute value for the $\boldsymbol{n}$ regions, and $\boldsymbol{w}_{i j}$ is the spatial weight matrix. The inference of significance is based on the permutation approach (Anselin, 1995). The results of the local Moran's statistics can be classified into four types of spatial association, and visualized in a LISA cluster map (Anselin, 1995).

\subsection{Data and Background}

The data for exploratory spatial data analysis has two types:

1. Regional per capita GDP and vital statistics data over the period 2001-2007 extracted from the Beijing area statistical yearbooks.

2. The administrative map of Beijing got from the administrative map of China with the scale of 1:1,000, 000 as shown in Figure 1.

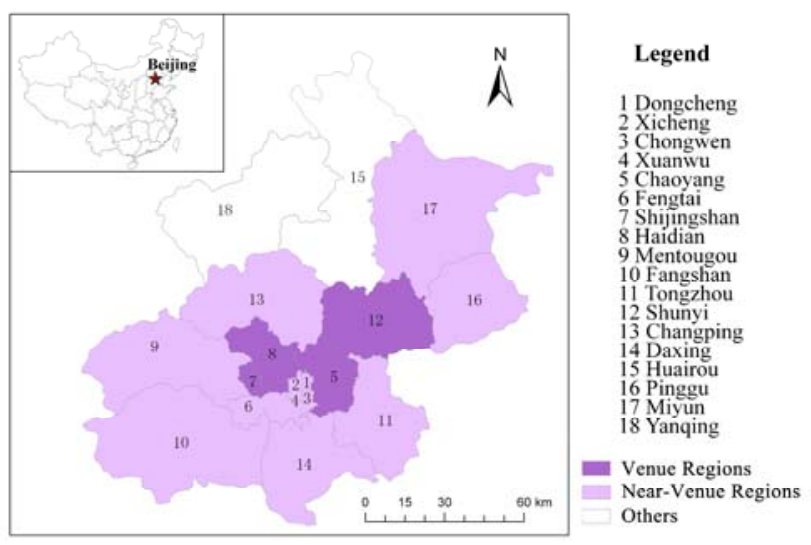

Figure 1. The administrative map of Beijing, China

The venues of Beijing Olympic were mainly distributed into four areas, one central area and three partitions. The central area is located in the Olympic park in Chaoyang district, and the three partitions are the university area, the western community, the northern scenic tourist area, located in Haidian, Shijingshan, and Shunyi respectively. Figure 1 depicts the administrative map of Beijing with the purple area as venue regions and the light purple areas as the regions adjacent to venue regions according to the spatial weight matrix.

The theory is that venue and near-venue regions would be positively impacted by the presence of the Olympic Games. The per capita GDP growth of near-venue regions might be more than those regions not close to Olympic events. Therefore, some changes in spatial patterns of regional economic disparities would be expected to be observed due to the influence of the Olympic Games. However, because of the neglect of spatial interactions and geographical location, the expected effects of the Olympic Games could be overestimated or underestimated by using the traditional statistics analysis. Therefore, in the next section, ESDA is adopted to investigate the influence of the Olympic Games for the development of regional economic, and reveal the spatial pattern of regional disparities in Beijing.

\section{RESULTS AND ANALYSIS}

In this paper, both global and local spatial autocorrelation analyses were carried out by using the GeoDa software package 
(Anselin et al., 2006). Figure 2 shows the global Moran's I statistic for per capita GDP from 2001 to 2007 using the spatial weight matrix with the threshold distance about 36,000 meters. The values of global Moran's I statistic show a downward trend from 2001 to 2007 and no significant positive or negative spatial autocorrelation, except for 2002. The results suggest that the locations of regions with high or low per capita GDP are almost random, and the spatial pattern has a developing trend of negative spatial autocorrelation since 2005.

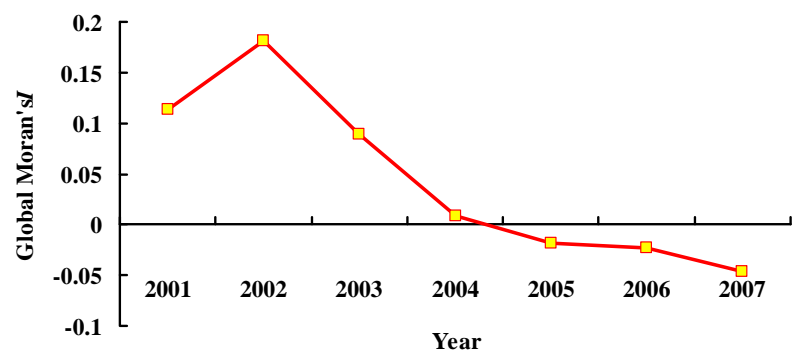

Figure 2. The chart of Moran's I statistics for per capita GDP of Beijing’s eighteen districts or county, 2001-2007

The Moran's $\boldsymbol{I}$ is a global statistic, and gives a single result to assess the spatial association pattern for the entire study area. Therefore, it cannot identify the regions contributing more to the results of global spatial autocorrelation, and detect the hot spot or atypical localizations. In order to solve these problems, the Moran scatter plot and LISA are adopted.

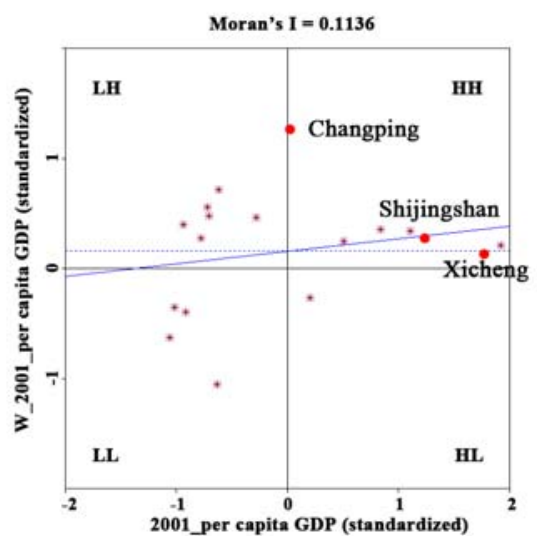

Figure 3. Moran scatter plot for per capita GDP, 2001

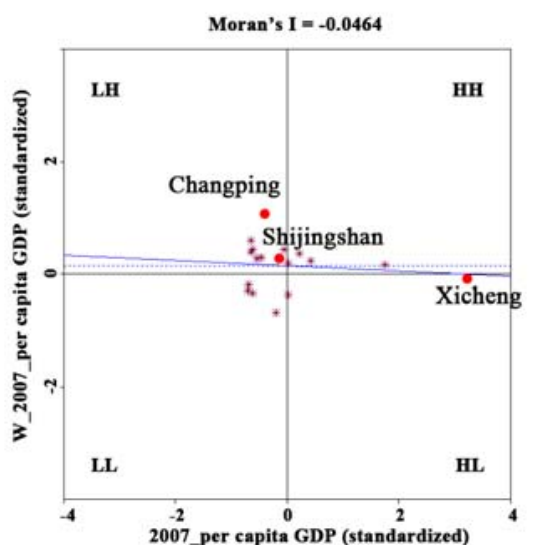

Figure 4. Moran scatter plot for per capita GDP, 2007
Figure 3 and 4 are the Moran scatter plots of per capita GDP for the initial year 2001 and final year 2007, respectively. It can be seen that 11 regions were characterized by positive spatial association (7 regions in quadrant $\mathrm{HH}, 4$ in LL) and 7 regions were characterized by negative spatial association (6 regions in quadrant LH, 1 in HL) in 2001. The regions of local spatial clusters with high values contributed more to the global spatial autocorrelation. However, 10 regions presented negative spatial association (8 regions in quadrant LH, 2 in HL) in 2007, which dominated the global spatial autocorrelation. The main change of spatial pattern between the two years concerned three regions In 2001, Xicheng, Changping and Shijingshan districts had similar high values of per capita GDP to their neighbours' and they were in quadrant HH. However in 2007, Xicheng located in quadrant HL indicating its higher level of economic development than their neighbours'. Contrarily, Changping and Shijingshan moved to the quadrant LH, which suggested their lower level of economic development comparing to their neighbours'.

The analysis of Moran scatterplots seems to be contradictory to the theory. Shijingshan District, as one of the venue regions, should have positive impact on the economic development. However, we can observe that it was relatively poorer than surrounding regions in 2007. Moreover, the other venue and near-venue regions have not obvious change in spatial pattern.
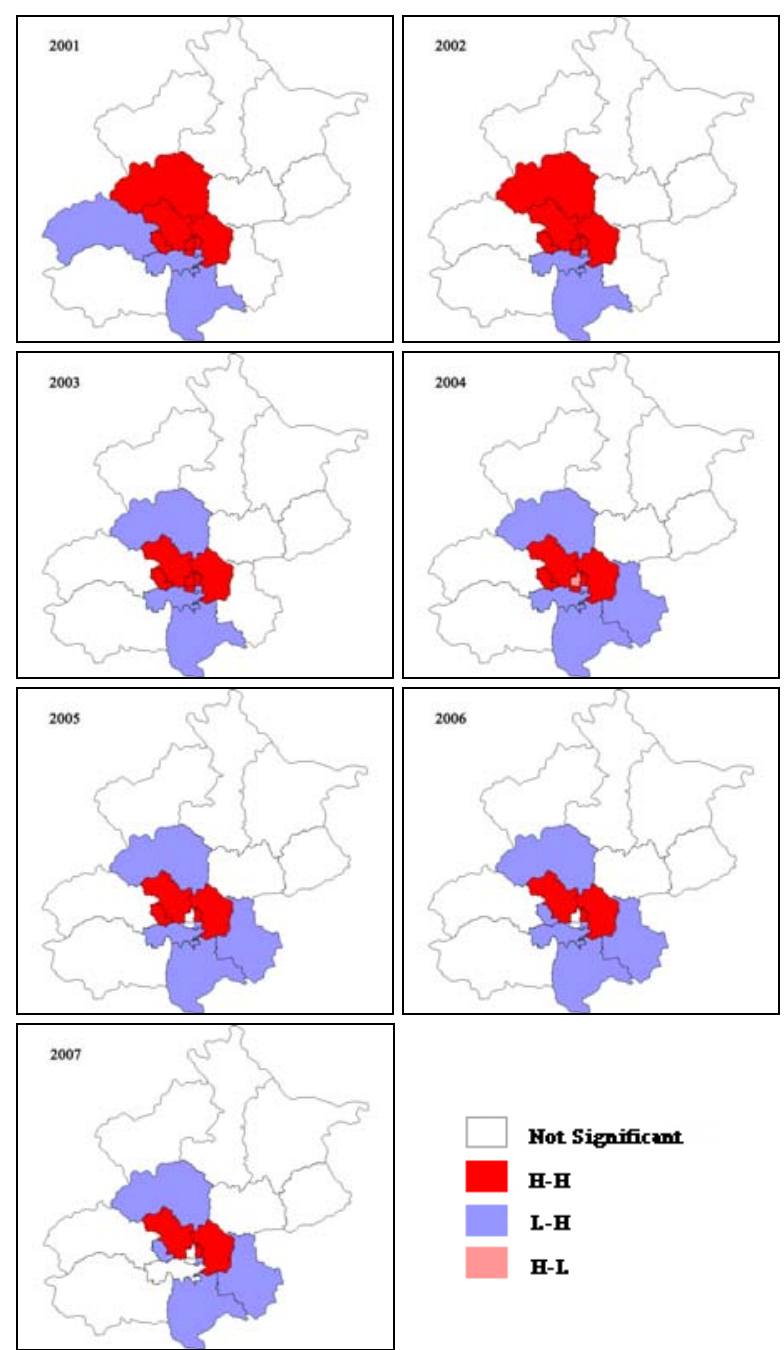

Figure 5. The LISA cluster map for per capita GDP of Beijing's 
Figure 5 is the LISA cluster map for per capita GDP from 2001 to 2007. The colored regions are significant with $\boldsymbol{p}=0.05$ for each year. The map indicated the two main patterns of spatial association, that is, clusters of high values surrounded by high values $(\mathrm{HH})$ and clusters of low values surrounded by high values (LH). Since 2003, Changping and Shijingshan had moved one after the other from $\mathrm{HH}$ to $\mathrm{LH}$ region, which caused a new centre-surrounded polarization scheme to gradually replace the North-South polarization scheme.

It is worth stressing the following interesting result: Although the Shijingshan is one of the venue regions, and Changping district is adjacent to both Haidian and Chaoyang (two venue regions), they belong significant to the LH quadrant lagging behind surrounded regions. It suggests the 2008 Olympic Games did not benefit the economic development of Shijingshan, and promote the efficient growth in per capita GDP of Changping district even adjacent to venue regions.

This result could be explained that the bid winning of 2008 Olympics accelerated the proceedings of Suburbanization of Residence in Beijing. The large bedroom communities such as Tiantongyuan and Huilongguan were built in Changping district, which led to the sharp increase of population migration from the city centre to Changping district. Although the 2008 Olympic Games promoted the growth in GDP of Changping district, its per capita GDP is much lower than neighbours' due to the increase of population. In addition, Shijingshan district also moved from the quadrant $\mathrm{HH}$ to the quadrant $\mathrm{LH}$ following Changping in 2006. An efficient cause should be the policybased limiting production of the Capital Iron and Steel Company. Due to the 2008 Olympic Games, Beijing accelerated the improvement of environment pollution by decreasing the production of the Capital Iron and Steel Company (Wan, 2006), which led to the lower GDP increasing in Shijingshan than some surrounding areas.

\section{CONCLUSIONS}

This paper attempted to investigate the influence of the 2008 Olympic Games for the development of regional economic disparities in Beijing since the year 2001 when Beijing won the bid. By combining ESDA with GIS technology, the spatial association patterns of regional per capita GDP were analyzed from 2001 to 2007.

The results showed not strong evidences of global spatial autocorrelation, but clear evidences of local spatial autocorrelation and spatial heterogeneity in the distribution of regional per capita GDP. Although the 2008 Olympic Games boost the economic development of Beijing, the total economic disparity in Beijing had not been alleviated.

Moreover, the results of the Moran scatterplot and the LISA highlight that a new centre-surrounding polarization scheme was gradually replacing the North-South polarization scheme in Beijing from 2001 to 2007.

\section{ACKNOWLEDGEMENTS}

This study was funded through support from a grant (Project Number: 2006AA120106) from the High-Tech Research and Development Programme of China, a grant from the State Key Laboratory of Resource and Environment Information System (Project Number: 088RA400SA).

\section{REFERENCES}

Anselin, L., 1994. Exploratory spatial data analysis and geographic information systems. New Tools for Spatial Analysis. Eurostat, Luxembourg, pp. 45-54.

Anselin, L., 1995. Local indicators of spatial associationLISA. Geographical Analysis, 27, pp.93-115.

Anselin, L., 1996. The Moran scatterplot as an ESDA tool to assess local instability in spatial association. Spatial Analytical Perspectives on GIS. Taylor \& Francis, London, pp. 111-125.

Anselin, L., 1999. Interactive techniques and exploratory spatial data analysis. Geographical Information Systems: Principles, Techniques, Management and Applications. Wiley, New York, pp. 251-264.

Anselin, L., Syabri I., Kho Y., 2006. GeoDa: An Introduction to Spatial Data Analysis. Geographical Analysis, 38, pp. 5 - 22.

Ertur C., Koch W., 2006. Regional disparities in the European Union and the enlargement process: an exploratory spatial data analysis, 1995-2000. Annals of Regional Science, 40, pp. 723765 .

Getis A., Ord J., 1996. Local spatial statistics: an overview. Spatial analysis: modeling in a GIS environment. Cambridge: Geoinformation International, pp. 261-277.

Le Gallo J., Ertur C., 2003. Exploratory spatial data analysis of the distribution of regional per capita GDP in Europe, 19801995. Regional Science, 82, pp. 175-201.

López-Bazo E, Vayá E, Artís M., 2004. Regional externalities and growth: evidence from European regions. Regional Science, 44, pp. 43-73.

Pu Y., Ge Y., Ma R., et al., 2005. Analyzing regional economic disparities based on ESDA. Geographical Research, 24(6), pp. $965-974$.

Qiu F., Zhu C., Tong L., et al., 2009. Spatial analysis of economic disparities of county level in Huaihai Economic Zone. Scientia Geographica Sinica, 29(1), pp. 56-63.

Wan X., 2006. Report on the implement of national economic and social development plan in 2006 and draft in 2007 of Shijingshan district.

http://huigu.bjsjs.gov.cn/affair/guihuajh/8a8481d21b533410011 b584631130012.html 